\title{
Fotografie e istituzioni museali: \\ il sistema della doppia copia \\ e l'accumulo dei fondi. \\ Le Regie Gallerie di Firenze, 1860-1906
}

\begin{abstract}
Beginning in the late 1850 s, photographic companies reproducing artworks kept in the Royal Galleries of Florence were required to submit copies of their work to the Ministry of Public Education and the museum's director. This paper shows that while photographers obeyed the rule, their photographs were stored away and left mostly unused for over two decades before the process of creating a proper collection began in the 188 os. Countering traditional histories of how photographic collections were constituted, this paper analyzes bureaucratic practices of documentation and questions the status of photographs in $19^{\text {th }}$ century museums.
\end{abstract}

\section{Keywords}

MUSEUM; HISTORICAL ARCHIVE; PHOTOGRAPHIC REPRODUCTIONS; PHOTOGRAPHIC COMPANIES; DEPOSIT; PHOTOGRAPHIC ARCHIVES; GALLERIE DEGLI UFFIZI; FLORENCE

D al 1867, anno della prima circolare nazionale riguardante i permessi concessi ai fotografi nei musei statali italiani, al 1902, anno della prima legge sul patrimonio artistico nazionale che regolamentava anche la riproduzione fotografica delle opere nelle collezione pubbliche, una richiesta costante caratterizzò i vari regolamenti volti a disciplinare la presenza dei fotografi nei musei italiani: lasciare almeno due copie positive di ogni negativa tratta, una destinata all'istituto dove la fotografia era stata realizzata e l'altra al Ministero della Pubblica Istruzione (d'ora in poi MPI) ${ }^{-1}$. Delle fotografie lasciate presso le Regie Gallerie di Firenze, oggetto di questo studio, sappiamo, ad esempio, che nel 1877 Carlo Brogi consegnò 16 fotografie; nel 1879 la 
Direzione delle Regie Gallerie ricevette 70 fotografie di vari fotografi; nel 1884 Vincenzo Paganori consegnò 214 riproduzioni fotografiche $\mathrm{e}$ gli Alinari 346; nel 1885 questi ultimi ne lasciarono, invece, 596 (fig. 1); nel 1889 Brogi consegnò alla Direzione 480 fotografie e sempre nello stesso anno la stessa ricevette 430 fotografie da vari fotografi (e la lista potrebbe continuare) ${ }^{-2}$.

Questo articolo ricostruisce la storia dei vari regolamenti volti a disciplinare la riproduzione fotografica di opere d'arte appartenenti allo Stato che furono emanati dal Ministero della Pubblica Istruzione in concordanza con la Direzione delle Regie Gallerie di Firenze, così da poter esaminare da una prospettiva inusuale il modo in cui le istituzioni considerassero le fotografie stesse. In particolare, l'articolo ricostruisce la pratica e le vicende intorno al lascito delle due copie dalla prospettiva di un'istituzione museale, quella delle Regie Gallerie di Firenze, utilizzando la copiosa e perlopiù inedita documentazione relativa alle riproduzioni fotografiche conservata presso l'Archivio Storico delle Gallerie Fiorentine (d'ora in poi ASGF) ${ }^{-3}$. Indagare il sistema del deposito è rilevante perché consente, da un lato, di esaminare le dinamiche del rapporto tra fotografi professionisti e musei e, dall'altro, di verificare in quale modo le fotografie siano entrate a far parte delle istituzioni museali e come siano state considerate e classificate. Su questo argomento generalmente si hanno poche informazioni perché, come hanno sottolineato Elizabeth Edwards e Christhoper Morton, le fotografie all'interno del museo hanno avuto e hanno uno status ambiguo, dato dalla loro natura e dalle varie funzioni affidate alla fotografia a seconda delle esigenze ${ }^{-4}$.

Che cosa venne fatto delle fotografie depositate? Quale status ricevettero? Erano documenti o veniva riconosciuto loro un altro valore? Furono sistematicamente collezionate o quale altro utilizzo fu previsto? E perciò quali erano le intenzioni nel richiederle?

Per quanto riguarda le fotografie che arrivavano al Ministero, recenti studi sull'Archivio Fotografico della Direzione Generale Antichità e Belle Arti, oggi assegnato all'Istituto Centrale per il Catalogo e la Documentazione e denominato Fondo Fotografico del MPI, rivelano che le copie d'obbligo, unite alle acquisizioni e alle campagne dirette, ne hanno costituito il fondo originario ${ }^{-5}$. In merito, invece, alle fotografie lasciate nelle istituzioni museali fiorentine, la questione è più complessa, anche perché se il Ministero chiese le fotografie con una maggiore consapevolezza dei loro possibili utilizzi per fini di ricerca, le istituzioni museali periferiche probabilmente soddisfacevano una richiesta che non coincideva necessariamente con le loro esigenze ${ }^{-6}$. Se fino agli anni Ottanta dell'Ottocento, infatti, negli Uffizi le fotografie venivano raccolte quasi involontariamente a seguito delle pratiche quotidiane dell'istituzione, negli anni Ottanta gli accumuli di fotografie cominciarono ad avere una certa consistenza, le richieste di riproduzione si moltiplicarono (esigendo pratiche di ordinamento) mentre contemporaneamente la storiografia dell'arte faceva sempre più riferimento al confronto visivo ${ }^{-7}$. Fu in questo periodo che i funzionari statali cominciarono a 


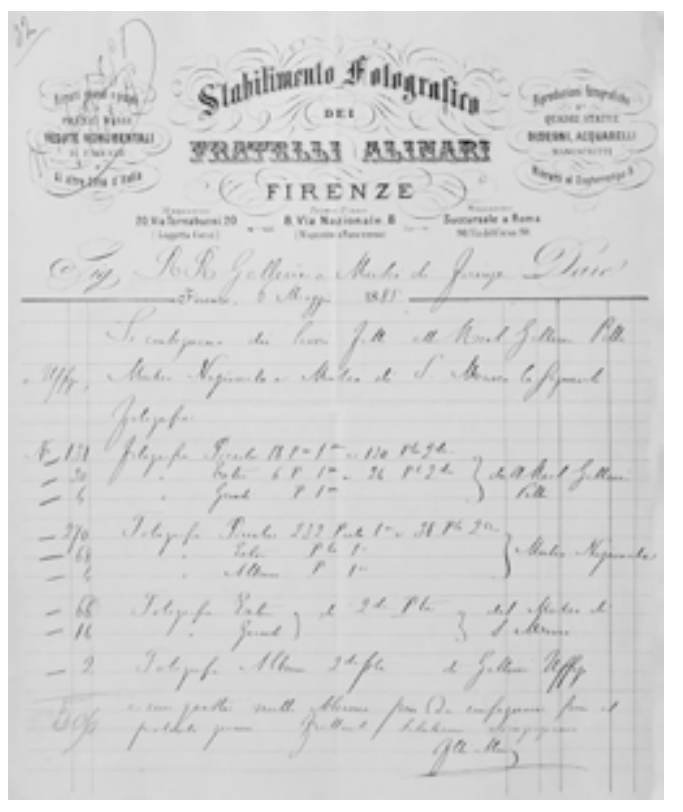

Stabilimento Fotografico

dei Fratelli Alinari,

Sig. RR Gallerie...,

lettera ms.,

Firenze, 6 maggio 1885.

Firenze, ASGF, 1885, J,

Affari Generali, 32.

Su concessione del

Ministero per i Beni

e le Attività Culturali

pensare di poter costituire una collezione fotografica che potesse essere utile agli studi; e fu allora che cominciò a prendere forma quello che potremmo definire il 'progetto Firenze'. Come dimostro nell'articolo, negli anni Ottanta dell'Ottocento una collezione di fotografie fu costituita presso il Gabinetto Disegni e Stampe ${ }^{-\mathbf{8}}$; agli inizi del Novecento fu poi fondato l'Archivio Fotografico degli Uffizi voluto dal direttore Corrado Ricci, archivio che doveva far parte di un particolare progetto legato alle collezioni grafiche che si concretizzò negli anni successivi negli Uffizi - ${ }^{9}$.

Le fotografie depositate dai fotografi professionisti costituirono, con un vero e proprio processo di sedimentazione, i nuclei originari di questi archivi ${ }^{10}$. A oggi, solo parte di queste fotografie è stata rintracciata nei diversi archivi esistenti presso i musei fiorentini. Alcune delle fotografie lasciate come copie d'obbligo sono state rinvenute nell'Archivio Storico delle Gallerie Fiorentine, altre ancora nell'Archivio Fotografico del Museo Archeologico di Firenze, ma molte altre attendono ancora di essere identificate ${ }^{-11}$.

\section{L'instaurazione del meccanismo del lascito della doppia copia (1860-1892) e le fotografie sepolte nell'Archivio della Direzione}

Nei documenti conservati nell'ASGF, il primo riferimento all'obbligo di lasciare una copia di ciascuna negativa tratta da un'opera conservata nelle Gallerie si trova nella corrispondenza del 1860, relativa alla richiesta dei fratelli Alinari di ottenere un permesso annuale per fotografare i quadri degli Uffizi; furono i fotografi stessi a obbligarsi "come di dovere a rilasciare alla Biblioteca della Galleria una copia di ciascun quadro ch'essi fossero per riprodurre" $-{ }^{12}$. Le parole degli Alinari fanno 
intendere che il lascito della copia fosse una pratica già comune nelle istituzioni museali e, infatti, già nel 1857 nell'Accademia di Belle Arti di Venezia e nell'Accademia di Brera ${ }^{-13}$ e nel 1858 nell'Accademia di Firenze ${ }^{-14}$, la copia veniva domandata ai fotografi che facevano richiesta di riprodurre opere ivi conservate. Nel 1860 e nel 1861 nelle Gallerie Fiorentine erano stati pubblicati Regolamenti per i copiatori, senza però riferimenti ai fotografi ${ }^{-15}$, e solo nel 1867 il Ministero inviò la prima circolare nazionale volta a uniformare le condizioni di accesso degli stessi nei musei ${ }^{-16}$. Fino a quella data, ogni galleria amministrava autonomamente la presenza dei fotografi e le regole a cui essi dovevano sottostare ${ }^{-17}$. Questo indica che la sistematizzazione della presenza dei fotografi nei musei non era esigenza primaria né del Ministero né della Direzione e che il lascito della copia non era condizione essenziale per lavorare nei musei.

D'altronde, le richieste di permesso erano poche - nelle Gallerie Fiorentine risultano essere meno di 25 tra il 1855 e il 1870 - e l'attività commerciale dei fotografi stava solo iniziando a porre alla Direzione nuovi problemi di amministrazione. Dai documenti fiorentini emerge con chiarezza che la fotografia, un'invenzione ancora recente "i cui risultati erano spesso fallaci" ${ }^{-18}$, veniva accolta nei musei con diffidenza durante quello che possiamo definire il primo periodo. La Direzione era preoccupata per la conservazione delle opere d'arte ma anche per la mediocrità delle riproduzioni fotografiche, così come per la concorrenza che le fotografie avrebbero alimentato rispetto al mercato delle copie, fonte di sostentamento per molti artisti dell'epoca. La diffidenza con cui la fotografia fu accolta nei musei non fu una caratteristica esclusivamente italiana, ma contraddistinse l'approccio anche di altri grandi musei europei: nel 1866, il permesso di riprendere fotografie al Louvre fu revocato fino a nuovo ordine perché l'attività dei fotografi era considerata pericolosa per le opere esposte ${ }^{-19}$.

Dopo questo primo periodo, a partire dal 1867, in Italia, le regole furono imposte a livello ministeriale in quelli che si possono individuare come due momenti distinti. In una prima fase, tra il 1867 e il 1893, il Ministero emanò, di concerto con la Direzione dei musei, una serie di regolamenti perlopiù rivolti ai copisti, con qualche articolo relativo ai fotografi. Una seconda fase, iniziata nel 1893 con l'emanazione del primo decreto esplicitamente dedicato alla riproduzione fotografica e culminata nel 1902 con l'emanazione della prima legge sul patrimonio artistico nazionale, fu caratterizzata da una chiara intenzione dello Stato di sfruttare il nuovo mercato delle immagini fotografiche di opere d'arte ${ }^{-20}$.

Per quanto riguarda la prima fase, nel 1867 il Ministero stilò il primo regolamento volto a uniformare le condizioni di accesso dei fotografi, nel 1876 fu inviata una circolare a tutti i musei riguardo la presenza dei fotografi, nel 1877 furono pubblicati un avviso (fig. 2) e un libretto dal titolo Norme da osservarsi per $i$ copiatori delle RR. Gallerie, nel 1879 venne pubblicato un nuovo avviso con lo stesso soggetto, nel 1881 un libello (fig. 3) e, infine, nel 1888 un nuovo libro ${ }^{-21}$. Fino al 1893, in 

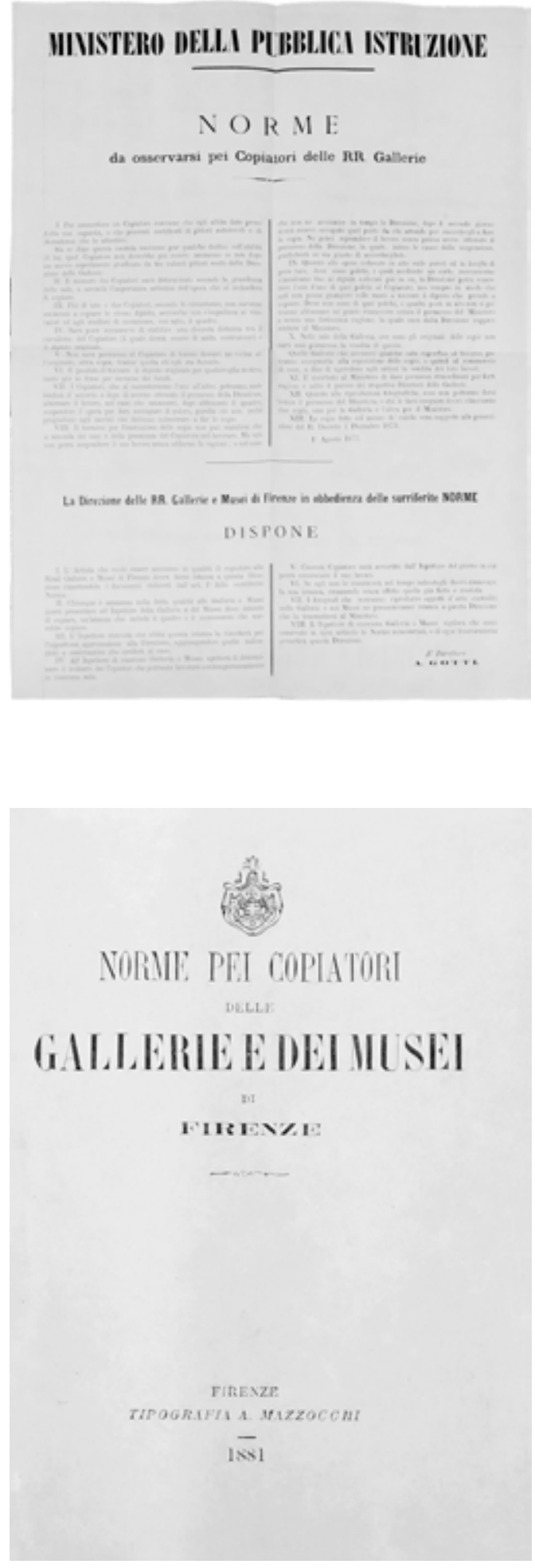

02

\section{Ministero della}

Pubblica Istruzione,

Norme da osservarsi

pei Copiatori delle RR.

Gallerie,

mat. a stampa, locandina

$36 \times 50 \mathrm{~cm}, 1$ agosto 1877 .

Firenze, ASGF, 1877, B,

Galleria delle Statue, 124.

Su concessione del

Ministero per i Beni e le

Attività Culturali

03

Norme pei Copiatori

delle Gallerie e dei Musei

di Firenze,

Firenze, Tipografia

Mazzocchi, 1881,

mat. a stampa, copertina.

Firenze, ASGF, 1881, B,

Galleria degli Uffizi, 97.

Su concessione del

Ministero per i Beni e le

Attività Culturali 
assenza di chiari riferimenti legislativi, la Direzione si riferì ad alcuni articoli di decreti, quali quello sui calchi delle opere d'arte del 1873 e, in alcuni casi, a quello per il servizio nei musei del 1877, che però non regolamentava specificatamente l'attività dei fotografi-22. Durante questa prima fase, i regolamenti erano spesso frutto di un confronto tra il Ministero e la Direzione dei musei, queste ultime davano indicazioni in base alle necessità delle proprie collezioni, come testimoniano sia la corrispondenza che, ad esempio, il regolamento del 1877 (fig. 2) nel quale sono indicate alcune norme aggiuntive specificatamente decise dalla Direzione delle Regie Gallerie di Firenze.

I regolamenti emanati fino al 1893 avevano le medesime finalità. Da un lato, il Ministero, di concerto con la Direzione fiorentina, intendeva affrontare questioni organizzative, di conservazione e di accesso: in nessun caso e per nessun motivo era possibile rimuovere dalla propria collocazione il dipinto da riprodurre; il permesso non sarebbe stato concesso se le macchine o gli sgabelli dei copisti avessero ingombrato il percorso dei visitatori; e per i fotografi, rispetto ai copiatori, era prevista una pratica più complessa, per la quale era necessario richiedere un permesso non solo alla Direzione, ma anche al Ministero ${ }^{-23}$. Dall'altro lato, con una norma dedicata espressamente alla riproduzione fotografica, fu stabilito il deposito obbligatorio delle due copie. Tale richiesta era stata spiegata nella circolare del 1876:

Affinché gli istituti traggano qualche vantaggio da tali concessioni, prego la signoria vostra di non dare per l'avvenire alcun simile permesso se non colla condizione che i fotografi rilascino due esemplari degli oggetti e monumenti fotografati $[\ldots] \mathbf{-}^{24}$.

$-$

Con l'emanazione della circolare divenne più costante il richiamo all'obbligo del deposito, anche a scapito di altre regole meno rispettate, come quella di spostare i dipinti. Nel 1878, ad esempio, i fratelli Alinari chiesero di poter spostare due quadri di Raffaello conservati a Pitti per poterli riprendere in una luce migliore; il Ministero, nel ricordare "l'obbligo ai fotografi di rilasciare gratuitamente due copie delle loro riproduzioni”, concesse il permesso in deroga ai regolamenti vigenti (fig. 4) ${ }^{\mathbf{2 5}}$.

D'altra parte, quella di spostare i dipinti era una necessità fondamentale per i fotografi d'arte e fu probabilmente per ottenere questo tipo di concessione che essi si adattarono a consegnare le copie richieste. Nonostante i sostanziali cambiamenti tecnici intervenuti tra gli anni Sessanta e Novanta - con novità quali l'introduzione delle lastre al collodio secche e al bromuro, che riducevano i tempi di esposizione da secondi a frazioni di secondi, di emulsioni ortocromatiche che permettevano di non invertire i toni e di apparecchi più precisi - fotografare i dipinti in un museo rimase sempre, ed è ancora oggi, un'operazione complessa ${ }^{-26}$. Per i fotografi era generalmente necessario muovere i dipinti, anche quelli di grandi dimensioni, per porli in luoghi rischiarati; utilizzando carrucole e scalei, collocavano i quadri su cavalletti di 


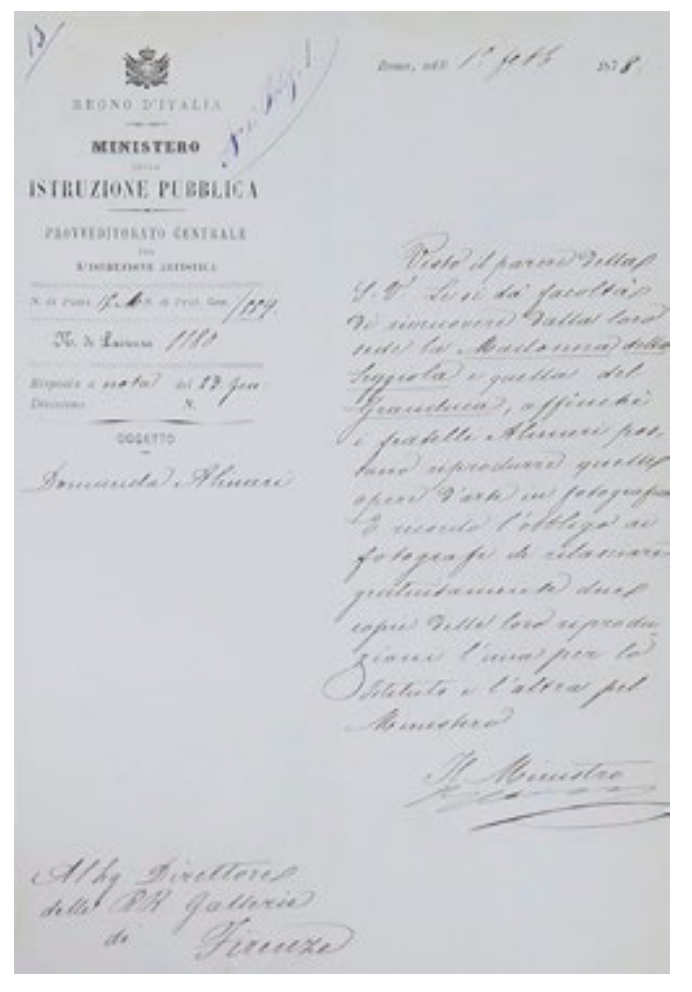

\section{Ministero della}

Istruzione Pubblica,

Visto il parere...,

lettera ms.,

Roma, 1 febbraio 1878.

Firenze, ASGF, 1878, A,

Galleria delle Statue, 13.

Su concessione del

Ministero per i Beni e le

Attività Culturali

fronte alle macchine fotografiche, modulavano l'illuminazione con riflettori di foglia d'argento e specchi e, nella fase di ripresa, utilizzavano diversi tipi di obbiettivi, differenti formati di lastre e, in fase di sviluppo e stampa, svariati materiali chimici e tecniche ${ }^{-27}$. La buona riuscita di una campagna fotografica presupponeva grande competenza e organizzazione e richiedeva di avere a disposizione molti materiali, oltre che tempo per ripetere anche numerose volte le operazioni. È anche per tali ragioni, quindi, che i fotografi depositavano, solitamente, le copie senza eccessive lamentele. C'è da aggiungere, inoltre, che il costo di due stampe era irrisorio rispetto ai profitti che i fotografi potevano trarre dalla riproduzione delle opere: all'inizio degli anni Settanta, gli Alinari affermano di lavorare "in media per lire 60.00o annue" -28 e nel 1873, come indicato nel loro catalogo, una fotografia piccola montata $(25 \times 18$ $\mathrm{cm})$ è in vendita a 1 lira e 75 cent., una fotografia mezzana $(35 \times 27 \mathrm{~cm})$ a 3 lire, una extra $(44 \times 33 \mathrm{~cm})$ a 5 lire e infine una grande $(60 \times 45 \mathrm{~cm})$ a 8 lire ${ }^{-29}$. Di conseguenza, anche se furono le istituzioni a imporre il lascito, gli editori fotografi, consapevoli del beneficio che poteva essere loro accordato e interpretando il deposito come uno scambio vantaggioso per le loro necessità, lasciarono generalmente le copie positive senza frequenti polemiche ${ }^{-30}$. Sebbene le lettere scambiate tra i fotografi, la Direzione e il MPI lascino supporre che, nella maggior parte dei casi, la pratica del lascito fosse ormai consuetudinaria, le prime conferme 
documentarie negli archivi degli Uffizi arrivano con la consegna di Giacomo Brogi del $1877^{-31}$. Quest'ultimo, nel consegnare 404 fotografie all'Accademia, ritiene "che la consegna di dette copie debba esimermi da farne il deposito che la legge richiede per assicurarsi la proprietà artistica" ${ }^{32}$. Brogi si riferisce qui alle tre copie che dovevano essere depositate alla Prefettura in osservanza della legge del 1865 sui diritti d'autore ${ }^{-33}$. Egli, perciò, sembra scambiare le due forme di deposito o forse accenna a una polemica riguardo alla quantità di copie che devono essere depositate.

Dalla corrispondenza tra il Ministero e la Direzione degli Uffizi emerge chiaramente come sia il primo a chiedere con insistenza che l'obbligo del lascito delle copie venga rispettato. Questa perseveranza può essere messa in correlazione con i progetti, attivati proprio intorno alla fine degli anni Settanta, che prevedevano l'utilizzo della fotografia come supporto all'inventariazione e alla catalogazione del patrimonio ${ }^{-34}$. Alla Direzione, invece, spettava principalmente il compito di gestire nei musei la presenza 'ingombrante' e quotidiana dei fotografi e dei copisti, i quali, per ragioni di studio e commerciali, si succedevano a ritmi serrati per poter riprodurre i grandi capolavori delle gallerie, dando vita, oltretutto, a varie querelles rispetto alla posizione (se nella prima, seconda o terza fila di fronte al dipinto) dei loro sgabelli. Questi sono alcuni dei motivi per cui, negli anni Sessanta e Settanta, la Direzione non curò in maniera particolare l'accumulo delle copie di deposito, con conseguenze dirette per il destino fisico delle fotografie. Nella loro richiesta del 1860, gli Alinari menzionano la Biblioteca in cui lasceranno le loro fotografie, ovvero quella degli Uffizi. Nel 1879, invece, Luigi Pigorini, allora Regio Commissario straordinario alle Gallerie e ai Musei di Firenze fa riferimento ad alcune fotografie conservate nell'Archivio della Direzione ${ }^{-35}$. In generale, tuttavia, le fotografie non erano conservate in maniera organizzata e non vi era alcun intento di collezionarle, come si evince in una corrispondenza del 1879 tra il MPI e il Regio Commissario. Il Ministero aveva chiesto al Commissario alcune fotografie, ma quest'ultimo dopo essersi informato sui prezzi, scriveva:

Quanto alla convenienza poi di comperarle per cederle in cambio di altre al governo dei Paesi Bassi, non io [sic] pronunziarmi favorevolmente. Nelle Gallerie di Firenze non esiste collezione di fotografie, né crederei utile di iniziarla. Non essendovi nelle Gallerie scuola di belle arti a cui quelle fotografie potrebbero giovare, resterebbero esse inutilmente sepolte nell'archivio, nella biblioteca della direzione ${ }^{-{ }^{36}}$. $-$

Benché il Commissario straordinario riconoscesse alle fotografie un ruolo per la formazione artistica, allineandosi così al pensiero dell'epoca e alla pratica sviluppatasi nel corso dei due decenni precedenti di costituire collezioni fotografiche presso le Accademie di Belli Arti (come dimostrato dai casi di Milano, Venezia e Firenze), nel caso delle Gallerie non trovava ragionevole avviare un simile progetto, affermando tra 
l'altro che se si fosse dovuto spendere del denaro, "credo valga meglio impiegarlo nel [...] mettere alla vista del pubblico quanto d'importante giace tutt'ora in stanze chiuse" - ${ }^{37}$. L'impegno, però, era stato preso e il Ministero aveva bisogno delle fotografie: l'affare si concluse poco dopo con la consegna di tre pacchi contenenti complessivamente 63 fotografie, ovvero "gli esemplari rimasti presso questa direzione secondo il disposto dell'art. XII delle norme ministeriali pei copiatori” ${ }^{\mathbf{3 8}}$.

Il fatto che, nel 1879, la Direzione avesse (o credesse di avere) solo 63 fotografie è un ulteriore indizio della mancanza, fino a quel momento, di uno specifico interesse nel collezionarle o conservarle, tanto che queste fotografie erano "just there" - ${ }^{39}$ : frutto delle prassi amministrative del museo, erano invisibili, scomparse, in una condizione che trova un corrispettivo nella loro situazione odierna, dal momento che a tutt'oggi la collezione non è stata localizzata.

\section{II primo utilizzo delle fotografie lasciate per deposito, 1883-1895}

Almeno fino alla fine del 1870, le fotografie depositate erano dunque sepolte nell'Archivio o nella Biblioteca della Direzione, senza che quest'ultima vedesse un'utilità nell'organizzarle e renderle consultabili. Questo modus operandi cambiò negli anni Ottanta. Nel maggio 1883 Nerino Ferri, conservatore del Gabinetto Disegni e Stampe degli Uffizi, informa il Direttore delle Regie Gallerie di aver compilato, come richiesto, una nota delle riproduzioni fotografiche ivi presenti ${ }^{-40}$. Si tratta della prima testimonianza di una raccolta di fotografie conservate presso il Gabinetto e di un utilizzo delle copie lasciate dai fotografi. È lo stesso Ferri a indicarcelo, quando spiega che le riproduzioni fotografiche saranno "riunite alle altre depositate presso codesta Direzione dai fotografi che man mano riproducono gli oggetti d'arte delle nostre gallerie” e serviranno

come la S.V. molto opportunamente deliberava, per fare dei baratti con altrettante fotografie riprodotte da oggetti d'arte che si ammirano in altri musei nostrani e forestieri. Provvedimento che porgerà modo agl'impiegati di questi istituti artistici di farsi almeno un'idea di molte pregevolissime opere d'arte esistenti in altre città, e che soltanto a pochi d'essi è dato poter visitare $-{ }^{41}$.

$$
-
$$

Era stato Cesare Donati, Direttore delle Regie Gallerie, a proporre nel 1881 al Ministero di realizzare un "album di fotografie" dei capolavori degli antichi maestri delle più importanti pinacoteche d'Italia $\mathrm{e}$ d'Europa, utilizzando le "fotografie che man mano si vanno accumulando in questa soprintendenza" in forza del nuovo regolamento e le copie giunte al Ministero dalle varie pinacoteche d'Italia ${ }^{-42}$. Queste fotografie sarebbero state messe a disposizione degli studiosi nelle Gallerie degli Uffizi e di Pitti, perché

Prima che ad abbellimento o a decoro, [...] le collezioni artistiche dovrebbero servire all'arte e allo studio di esse [...]. Siffatta raccolta gioverebbe 
Nerino Ferri,

Nota delle Riproduzioni..., lettera ms.,

Firenze, 8 maggio 1883.

Firenze, ASGF, 1883, D,

Galleria degli Uffizi, 62.

Su concessione del

Ministero per i Beni e le

Attività Culturali

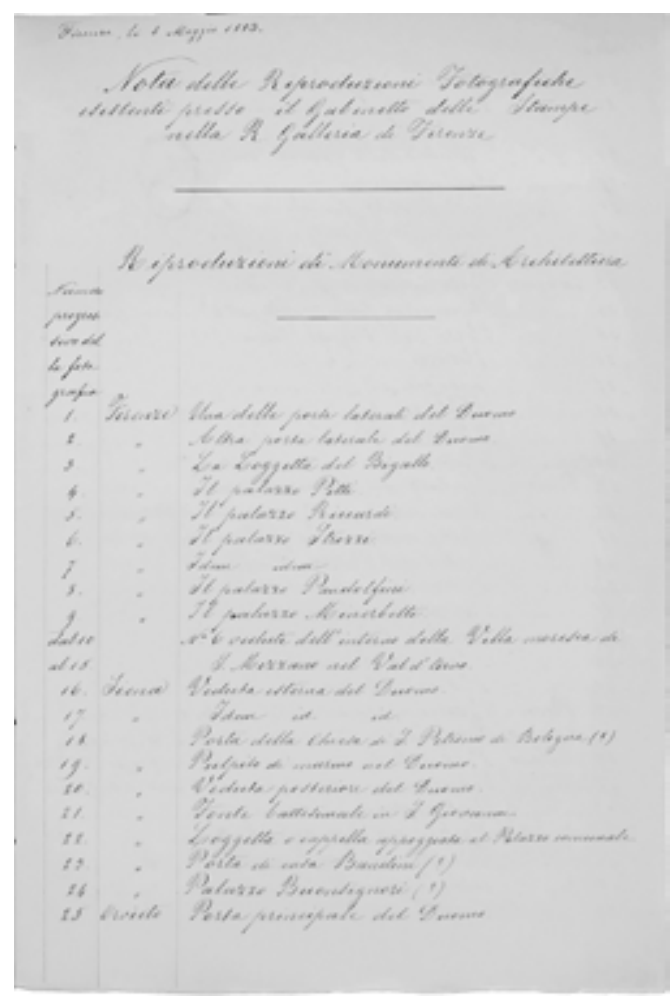

pure a questa amministrazione, la quale spesso si trova a dover dar giudizio di opere d'arte conservate in gallerie e musei stranieri ${ }^{-43}$.

Dopo che il Ministero aveva accolto la richiesta, si può supporre poiché non sono state al momento rinvenute lettere tra Ferri e Donati fra il 1881 e il 1883 - che al conservatore fosse stato affidato il compito di sovrintendere alla costituzione di questo album, inventariando le fotografie esistenti. A una lettera del maggio 1883, Ferri allega infatti una "Nota delle riproduzioni fotografiche esistenti presso il Gabinetto delle stampe" in cui si contano un totale di 363 fotografie, a cui si aggiunge la raccolta di 1.487 fotografie dei disegni degli Uffizi realizzati dalla ditta Braun, conteggiata separatamente (fig. 5). La corrispondenza tra Ferri e la Direzione attesta che la collezione di fotografie conservata presso il Gabinetto delle stampe, identificata come tale fin dal 1884, crebbe rapidamente: nell'agosto 1883 le fotografie erano 710, mentre nel 1884 ne furono registrate prima 840 e poco dopo $1.019^{-44}$. Nella nota del 1887 i numeri progressivi assegnati alle fotografie vanno dal numero $1.772 \mathrm{al}$ 1.903, mentre nel 1890 dal numero 1.904 al 2.333, per un totale, solo in quest'ultima tranche, di 434 fotografie inserite ${ }^{-45}$. La provenienza delle fotografie non sempre è rintracciabile, perché nelle note compilate da Ferri vengono indicati solo il soggetto e il luogo, solo in alcuni casi il nome dell'autore della fotografia. Nella maggior parte dei casi, ad ogni 
modo, le fotografie erano le copie d'obbligo, come confermano le molte lettere di Ferri presenti in Archivio. A titolo esemplificativo, la quarta appendice alla nota del 1883, compilata nel 1884, coincide con le 173 fotografie inviate dagli Alinari in doppio esemplare.

L'ultima nota scritta da Ferri è del 1890, anche se i documenti indicano che egli continuò a ricevere fotografie anche in seguito. Nel 1892 il conservatore scrisse alla Direzione per accusare ricevuta di 52 fotografie da parte della ditta Brogi, sottolineando che "le dette riproduzioni hanno preso i numeri dal 4.599 al 4.650 della raccolta di fotografie custodite in questo Gabinetto" - ${ }^{46}$. La presenza di una collezione di fotografie presso il Gabinetto è poi confermata sia nel 1893 sia nel 1894 da documenti che indicano che esse arrivavano anche attraverso dono o acquisto diretto da parte di Ferri.

Nel 1895 il Direttore degli Uffizi Enrico Ridolfi proponeva ai fratelli Alinari e alla ditta Brogi un nuovo progetto:

Questa Direzione reputerebbe necessario l'impianto di una regolare amministrazione relativa alle riproduzioni fotografiche che essa richiede ai signori fotografi o commette loro di eseguire per i suoi studi, nonché di quelle che i signori fotografi stessi sono in dovere di dare al seguito dei permessi che da essi ottengono per lavorare negli Istituti ${ }^{47}$.

Questa amministrazione sarebbe servita a gestire il dare e l'avere fra la Direzione e i fotografi e, continuava Ridolfi,

\section{$-$}

La Direzione per i suoi studi ha di frequente bisogno di procurarsi delle riproduzioni fotografiche di oggetti d'arte eseguite dai vari stabilimenti fotografici in varie parti d'Italia e d'altro lato gli stabilimenti medesimi avendo frequente bisogno di eseguire riproduzioni negli istituti [...] si trovano a dover dare alla Direzione le copie di tali riproduzioni prescritte dal vigente regolamento ${ }^{-48}$.

-

Sulla base di queste premesse, viene proposto agli stabilimenti fotografici di consegnare alla Direzione, "invece delle riproduzioni dovute, altre che potrebbero abbisognare per i suoi studi" -49 .

Nei documenti dell'ASGF degli anni successivi non si fa più alcun riferimento alla costituzione di questa amministrazione; l'assenza di documenti potrebbe essere motivata dalla mancata continuazione del progetto, ma è anche ascrivibile a una forte diminuzione dei documenti relativi alla riproduzione fotografica dal 1895 in poi. Anche i riferimenti alla collezione di fotografie diminuiscono e, soprattutto, non ci sono più le note manoscritte da Ferri. Tutti questi fattori non consentono di ricostruire con chiarezza le vicende successive di questa collezione ${ }^{-50}$. A ciò va aggiunto che la localizzazione odierna di queste fotografie rimane ignota. Miriam Fileti Mazza ha affermato che esse costituiscono la base della Fototeca conservata nel Gabinetto Disegni e Stampe ${ }^{-51}$. Tuttavia la Fototeca conserva solo fotografie di disegni, ponendo un 
interrogativo sull'ubicazione delle fotografie di dipinti e sculture lasciate come deposito. È plausibile, quindi, che le fotografie, considerate solo come un supporto alle attività di studio, circolassero nei vari ambienti degli Uffizi senza trovare una stabile collocazione ${ }^{-52}$.

Rintracciare e analizzare questo corpus consentirebbe oggi di fornire indicazioni importanti sulla sua costituzione. In ogni caso è rilevante sottolineare che tale collezione venne effettivamente costituita e che questo progetto è ricollegabile, anche se solo per precocità di nascita, con il Ricetto fotografico dell'Accademia di Brera del 1899. In quest'ultimo archivio - che Corrado Ricci, uno degli ideatori, proporrà poi a Firenze nel 1903 - sarebbero state raccolte non solo riproduzioni di opere d'arte, ma anche fotografie etnografiche, storiche, di luoghi, avvenimenti, arti minori, persone ragguardevoli utile agli studi ${ }^{\mathbf{5 3}}$.

Le fotografie lasciate per deposito costituirono un nucleo sostanziale della collezione e del progetto proposto da Ridolfi, il quale nella lettera inviata ai fotografi scriveva che l'idea di costituire l'amministrazione era conseguenza dell'incremento delle fotografie che giungevano al museo quasi sicuramente dovuto all'emanazione, nel 1893, del primo decreto esplicitamente dedicato alla riproduzione fotografica delle opere appartenenti allo Stato ${ }^{-54}$. L'aumento delle fotografie era anche ascrivibile alla democratizzazione della pratica fotografica, dovuta principalmente ai cambiamenti tecnologici che permettevano (quasi) a tutti di fare fotografie, con un conseguente incremento esponenziale delle richieste di permesso. I dilettanti, inoltre, come espressamente dichiarato dal Ministro, dovevano seguire le stesse regole imposte ai fotografi professionisti ${ }^{-55}$. Il Ministero intendeva fornire le medesime norme a tutti i musei italiani, regolamentando in maniera più stringente la presenza dei fotografi. La richiesta di permesso diventava ora più precisa $\mathrm{e}$ richiedeva di indicare lo scopo della ripresa, il metodo da applicare, la presunta durata dell'operazione e il nome dell'operatore (art. 2); in una serie di articoli si regolamentavano invece le questioni conservative, che imponevano di non movimentare i quadri esposti (art. 5). L'aspetto più rilevante, però, riguardava l'obbligo dei fotografi di consegnare due copie positive e una negativa per i cimeli scientifici e letterari e tre copie positive per i monumenti e i cimeli artistici (art. 3).

Il decreto del 1893 rappresentava perciò l'apice di una politica ministeriale che, fin dal 1867, aveva avuto come elemento ricorrente la richiesta delle copie fotografiche (ora integrata dall'obbligo di consegnare, in aggiunta alle riproduzioni eventualmente ritoccate, anche gli esemplari senza ritocco). Il decreto, che rientrava in una maggiore consapevolezza ministeriale degli utilizzi della fotografia, era stato motivato anche dalla necessità di provvedere alla migliore preparazione del catalogo generale dei monumenti e degli oggetti d'arte del Regno ${ }^{-56}$ : proprio nel 1892, a Roma, era stato istituito l'ufficio, che diventerà in seguito il Gabinetto Fotografico Nazionale, che avrebbe avuto il compito di coadiuvare la realizzazione del catalogo ${ }^{-57}$. A ciò si aggiungeva la possibilità di uno sfruttamento economico: nel disegno di legge sulla 
conservazione dei monumenti del 1892, il Ministero aveva previsto infatti una tassa tra L. 10 e L. 50 per la riproduzione fotografica di opere conservate nei luoghi chiusi di proprietà statale, così da far fronte ai problemi finanziari creati dalle esigenze di tutela ${ }^{-58}$. L'articolo fu poi abrogato grazie anche all'interessamento dei fotografi, in particolare di Carlo Brogi e della Società fotografica italiana $-{ }^{59}$.

Il decreto del 1893 destò le critiche delle aziende fotografiche. Vittorio Alinari, in un già noto libello, lo definì "illegale ed incostituzionale perché arbitrario e assurdo" - ${ }^{60}$. Alinari criticava la tipologia di informazioni che dovevano essere inserite nella domanda, ritenendole espressione della limitata competenza dei compilatori del regolamento, ma dissentiva soprattutto nei confronti dell'onere imposto alle case fotografiche di depositare un così alto numero di copie ${ }^{-61}$. Nonostante queste critiche, negli anni Ottanta e Novanta i fotografi continuarono a inviare le fotografie, il cui numero, rispetto ai decenni precedenti, aumentò esponenzialmente. Il fatto che ai fotografi venisse ora richiesto di montare direttamente le copie su cartoncino suggerisce una nuova volontà di conservare, archiviare, titolare e utilizzare le fotografie depositate.

Un episodio avvenuto sul finire degli anni Ottanta risulta estremamente indicativo dei rapporti tra la Direzione e i fotografi. Nel 1887 la ditta alsaziana Adolphe Braun aveva richiesto di fotografare tutta la collezione di Palazzo Pitti e degli Uffizi, con una serie di concessioni straordinarie volte a facilitare le operazioni. Tra queste vi era la richiesta di costruire un atelier provvisorio in un luogo ben rischiarato quale "una terrazza come quella che dagli Uffizi dà sulla Piazza della Signoria” -62 dove, durante la stagione propizia, avrebbe portato le opere dei due musei per poterle riprendere. La Direzione, seguendo le insistenze del Ministero, concesse la costruzione dell'atelier richiedendo anche l'edificazione di un padiglione per riparare i quadri dalle intemperie e dai raggi solari, "potendosi dare il caso che un quadro di grandi dimensioni debba rimanere per più ore esposto sulla terrazza perché appunto per la sua mole deve essere spostato da più persone" ${ }^{63}$. A quel punto, la ditta Brogi polemizzò fortemente per le concessioni accordate a Braun, una ditta straniera con la quale gli stabilimenti fotografici italiani erano in competizione nel mercato europeo. Viste le insistenze, sia ad Alinari che a Brogi fu dunque concesso di fotografare nuovamente tutta la collezione di Pitti e degli Uffizi e di costruire - anch'essi - un atelier sulla terrazza per poter, come affermarono i fotografi nelle loro richieste, stare al passo con le nuove invenzioni ${ }^{-64}$. Come riporta con preoccupazione il conservatore della Galleria Pitti, Eugenio Pieraccini, Brogi e Alinari, in deroga ai regolamenti vigenti, poterono staccare i quadri dalle pareti e spostarli - anche quelli di grandi dimensioni - sulla terrazza degli Uffizi, dove venivano lasciati anche per tutto il giorno in attesa di essere posti sul cavalletto per essere riprodotti ${ }^{65}$. Come è possibile rilevare dalle più di cento lettere in cui si affronta la questione, Alinari e Brogi, come contropartita a tali concessioni, dovettero pagare il personale del museo e il conservatore per il lavoro straordinario, lavorare solo negli 
orari di chiusura delle gallerie (la mattina molto presto e a fine giornata) e lasciare le copie richieste. Episodi come questi, per quanto eccezioni, fanno ben capire quanto il lascito delle due copie fosse per i fotografi un'ottima contropartita a fronte di un'interpretazione spesso di favore delle norme vigenti. La situazione, però, cambiò drasticamente con l'attuazione della prima legge sul patrimonio artistico nazionale.

\section{La prima legge sul patrimonio artistico nazionale e il primo progetto di Archivio Fotografico (1902-1906)}

Nel 1902 venne emanata la prima legge sul patrimonio artistico nazionale, mentre nel 1904 divenne operativo il regolamento per la sua attuazione ${ }^{-66}$. Nove articoli della legge reiteravano le disposizioni già previste dai regolamenti precedenti in merito alle riproduzioni fotografiche, con due eccezioni. L'articolo 246 prevedeva il pagamento di una tassa, variabile tra L. 1 e 10 a seconda dell'importanza dell'oggetto fotografato; l'articolo 249 stabiliva l'obbligo di consegnare una negativa perfetta e senza ritocco e due copie positive, aggiungendo che "le negative consegnate rimangono di proprietà dello Stato, che potrà servirsene ai fini della riproduzione con altri mezzi fotomeccanici" ${ }^{67}$.

Durante l'interpellanza parlamentare presentata dall'onorevole Filippo Torrigiani al Ministro della Pubblica Istruzione “per sapere perché con una circolare abbia sospeso i permessi per riproduzioni fotografiche delle opere d'arte e monumenti dello Stato" il 13 giugno 1904, -68 Emilio Pinchia, sottosegretario di Stato per l'istruzione pubblica, aveva sostenuto che se le concessioni date ai fotografi avevano rappresentato fino ad allora una forma di privilegio, da quel momento esse avrebbero dovuto tramutarsi in un profitto per lo Stato ${ }^{-69}$. La tassazione sulla riproduzione era intesa, infatti, a costituire una cassa destinata all'acquisto di opere, mentre i negativi sarebbero stati usati per la catalogazione del patrimonio artistico nazionale, compito già affidato al Gabinetto Fotografico di Roma. Oltre alla tassa e al lascito dei negativi, nel maggio del 1904, poco prima dell'emanazione del regolamento, il Ministero decise inoltre di sospendere i permessi ${ }^{-70}$.

La decisione di trarre un profitto dalla riproduzione fotografica di opere appartenenti allo Stato non fu solo italiana. In Francia, nel 1885, la ditta Braun firmò una convenzione con lo Stato che la portò a ottenere un atelier di riproduzione fotografica all'interno del museo, una sala di esposizione e una di vendita e il titolo di fotografo ufficiale del Louvre. In cambio, i negativi sarebbero diventati di proprietà dello Stato, con l'impegno di consegnarne un minimo di 7.000. Anche in Francia, come in Italia, i fotografi reagirono con forza alla ratifica di questo contratto, che videro come l'instaurazione di un monopolio di fatto ${ }^{-71}$.

In relazione alle delibere del Ministero del 1904, la reazione dei fotografi non si fece attendere. Fu Carlo Brogi, appoggiato dalla Camera di Commercio di Firenze e dalla Società Fotografica Italiana, con sede in città, a guidare la protesta, affermando che l'applicazione di questi articoli sarebbe stata esiziale per l'industria fotografica italiana ${ }^{-72}$. La 
richiesta principale fu quella di revocare l'obbligo di lasciare le negative poiché, così facendo, lo Stato avrebbe legalizzato la pratica di utilizzare le fotografie senza pagare agli autori alcun diritto di riproduzione, eludendo così la proprietà artistica ${ }^{-73}$. Il mancato rispetto di questo diritto era al tempo una pratica comune, considerando che, negli stessi anni, le tre maggior aziende fotografiche in Italia - Alinari, Brogi e Anderson - avevano inviato una circolare ai loro clienti e rivenditori per tutelarsi contro l'utilizzo da parte degli editori delle loro fotografie senza il pagamento dei diritti $-{ }^{74}$. È importante sottolineare che commentando la richiesta dei negativi, alcuni adombrarono il timore che lo Stato avrebbe potuto commerciare nuove stampe tratte da essi. Giovanni Santoponte sottolineò che non era in discussione il diritto dello Stato di realizzare riproduzioni delle proprie opere e di metterle in circolazione in concorrenza con quelle dei fotografi commerciali, "come, in piccola scala, fa già da tempo"; tuttavia, aggiungeva, non si può ammettere che lo Stato stesso

mentre protegge il fotografo dalle contraffazioni, per parte di concorrenze sleali, dei lavori eseguiti, accettandone il deposito ai sensi della legge sulle opere dell'ingegno, si arroghi il diritto di profittarne egli stesso dell'opera altrui imponendo perfino la consegna dei mezzi per sfruttarla più facilmente ${ }^{-75}$.

Similmente, Carlo Brogi faceva riferimento a

un deposito dello Stato [in cui] chiunque possa (non pagando che le spese o poco più) andare a chiedere tutte le positive che vuole siano per proprio conto tratte da quella negativa e poi farne l'uso che vuole. Ma questa è pirateria di terra! II fotografo dovrebbe eseguire riproduzioni per lo Stato e lo Stato metterle a disposizione di tutti! II frutto dell'ingegno o del semplice lavoro è proprietà privata inviolabile ${ }^{-76}$.

Come si evince da una lettera finora inedita di Brogi a Corrado Ricci - all'epoca direttore delle Regie Gallerie - del 26 dicembre 1905, nel luglio del 1904 i fotografi avevano avviato un'opera di boicottaggio delle opere appartenenti allo Stato ${ }^{-77}$. Questa azione, spiegava Brogi, non arrecava danno alle ditte fotografiche ma alla cultura in generale, dato che, fino a quel momento, erano stati gli stabilimenti fotografici

a scialacquare con la propria tasca per fare riproduzioni che tornavano d'utilità agli studi, ma non avevano sufficiente valore remunerativo sotto l'aspetto commerciale $-{ }^{78}$.

Ora, proseguiva la lettera, si sarebbe tornati al paesaggio e alle vedute, soggetti liberi sui quali il governo non avrebbe potuto intervenire e che avrebbero garantito maggiore facilità di vendita "nella massa della gente”. Brogi consigliava al Ministero di valutare quali fossero stati gli 
effetti reali del regolamento, dal momento che aveva bloccato la realizzazione di nuovi negativi. Nei documenti non è conservata nessuna risposta diretta, ma se ne può trovare una indiretta nel decreto emanato nel 1906 - quando Ricci era alla Direzione Generale delle Antichità che modificava il regolamento: le tasse da pagare erano state abbassate tra L. 0,50 e 5 e, soprattutto, non veniva più richiesto di lasciare i negativi ma solo le tre copie positive di ogni negativa originale ${ }^{-79}$.

Questi eventi devono essere collegati con l'istituzione di un archivio fotografico presso gli Uffizi: una raccolta pubblica in una sede pubblica, come scrisse Ricci in un già noto appello del 1903, dove tutti avrebbero potuto consultare i documenti utili per i propri studi, istituita grazie alle donazioni e alle fotografie depositate per legge ${ }^{-\mathbf{8 0}}$. Ad oggi non sono state individuate tracce di questo archivio; in ogni caso la sua definizione, di cui qui si può solo accennare brevemente, sembra essere più complessa di quanto solitamente supposto. Mi riferisco in particolare all'ipotesi secondo la quale l'appello di Ricci portò alla costituzione del Gabinetto Fotografico nel $1904^{-81}$. Il Gabinetto Fotografico (tutt'oggi esistente) sembra essere stato una realtà separata dall'Archivio, e fu stando a un documento d'archivio inedito che contraddice, ciò che è stato precedentemente affermato da Marilena Tamassia che ha collocato la nascita del Gabinetto al 1904 - costituito nel 1906 per gestire le richieste di riproduzioni di opere esistenti nelle Gallerie ${ }^{-{ }^{82}}$.

I documenti dell'ASGF attestano che Ricci, alla Direzione Generale Antichità e Belle Arti, aveva previsto l'apertura di un archivio fotografico come già evidenziato nell'appello del 1903, e l'archivio avrebbe fatto parte di un particolare progetto museografico per le collezioni grafiche degli Uffizi di cui rende conto, in prima istanza, Ferri nel 1908. Quest'ultimo, infatti, fa riferimento al "Gabinetto Disegni e Stampe", a una "Raccolta Storico-Topografica fiorentina", a una "Raccolta Iconografica”, a una "Biblioteca Artistica" e, non ultimo, a un "Archivio Fotografico" - ${ }^{83}$. Pochi anni più tardi, nel 1911, Alberto Parisotti, nuovo direttore delle Gallerie, nel riassumere la struttura gestionale di alcuni dipartimenti riferisce nuovamente dell'esistenza di un "Archivio Fotografico", scrivendo che esso consta di circa 20.000 fotografie e che è articolato in due sezioni: quella artistica, costituita da 15.000 fotografie divise per autori e per scuole, e quella topografica; oltre a ciò, egli segnala la presenza di 2.000 negativi che "questo Gabinetto Fotografico ha tratto" - ${ }^{84}$. All'Archivio Fotografico, come scrive Parisotti, si aggiungeva poi la "Collezione iconografica", costituita da circa 40.000 tra incisioni e fotografie, ma priva di un adeguato ordinamento e in alcune parti senza una netta distinzione con l'Archivio Fotografico, data forse da una vicinanza fisica delle fotografie che aveva portato a creare confusioni tra le due raccolte.

La costituzione di un archivio fotografico che potesse avere una sede pubblica presso gli Uffizi rappresenta l'atto finale di un processo iniziato negli anni Ottanta, quando Ferri scrisse la sua prima nota sulle riproduzioni fotografiche collocate presso il Gabinetto Disegni 
e Stampe. La ricerca non ha individuato fisicamente le collezioni, ha invece individuato la documentazione scritta relativa a questa serie di collezioni fotografiche costituite nel tempo presso gli Uffizi: una presso il Gabinetto Disegni e Stampe, una connessa al progetto presentato da Ridolfi nel 1895, infine l'archivio voluto da Ricci. Invece di tracciare la genesi di queste collezioni, ho considerato qui il dispositivo legale che ne ha permesso la costituzione e, così facendo, è stato possibile evidenziare che le copie d'obbligo - frutto delle prassi regolamentari del museo, non acquisite secondo un progetto specifico - costituirono una parte importante di questi primi nuclei formatisi presso le Regie Gallerie Fiorentine. Queste fotografie, come testimoniano i documenti, furono inizialmente lasciate su uno scaffale della biblioteca, poiché evidentemente mettevano in discussione le categorie e i valori assodati che presiedevano alla costituzione di una collezione museale. Solo successivamente esse poterono cambiare status, diventando documenti in grado di coadiuvare la gestione del patrimonio e l'avanzamento degli studi storico-artistici.

A oggi, lo stato delle conoscenze sull'argomento è estremamente parziale. Molte di queste fotografie non sono state individuate e non siamo a conoscenza dell'esatta collocazione dell'Archivio Fotografico e delle fotografie inventariate da Ferri. Presumibilmente le collezioni furono smembrate: alcune si trovano nei musei nei quali sono conservate le opere riprodotte nelle fotografie; altre nel Gabinetto Disegni e Stampe e nell'Archivio Storico delle Gallerie Fiorentine. Altre, invece, attendono ancora di essere identificate, a ricordarci che lo status delle fotografie nei musei, quando non si tratti delle cosiddette fotografie artistiche, è ancora molto incerto e complesso ${ }^{-85}$.

\author{
L'articolo, redatto \\ nell'estate del 2019, ha \\ trovato conferma, in \\ alcune sue ipotesi, nel \\ ritrovamento da parte \\ della Soprintendenza del \\ Regio Archivio Fotografico \\ degli Uffizi. L'Archivio è \\ attualmente oggetto di un \\ progetto d'inventariazione \\ supervisionato dalla \\ dott.ssa Valentina \\ Conticelli. \\ $-{ }^{1}$ I regolamenti non \\ sono mai stati l'oggetto \\ principale degli studi.
}

Piuttosto, la loro citazione
è stata funzionale a
tracciare la genesi di una
collezione o il rapporto
tra una particolare
istituzione e la fotografia
o il lavoro di alcuni
fotografi. Inoltre, almeno
fino al 1893, la situazione
a livello nazionale era
molto disomogenea,
rendendo i vari contributi
sull'argomento parziali
e incompleti. Vedi, fra
i testi che accennano a
più di un regolamento:
Maffioli 2017, pp. $340-341$

Piuttosto, la loro citazione tracciare la genesi di una collezione o il rapporto tra una particolare istituzione e la fotografia (licuni fotografi. Inoltre, almeno fino al 1893, la situazione a livello nazionale era molto disomogenea, rendendo i vari contributi sull'argomento parziali i testi che accennano a Maffioli 2017, pp. 340-341

\footnotetext{
e Migliorini 1994, pp. 45-47 sull'Accademia di Belle Arti di Firenze; Filippin 2015, pp. 11-13 sull' Accademia di Belle Arti di Venezia; Berardi 2014, pp. 184-185 sul Fondo Fotografico del MPI; Ghibaudi 2012, p. 427 e Strambio 2000, pp. 30-31 sull'Accademia di Belle Arti di Brera; Arbeid 2015, p. 3 sul Museo Archeologico di Firenze; Binazzi 2020 sulle Gallerie degli Uffizi. Dal punto di vista dei fotografi l'argomento è stato accennato in Maffioli 2014,
} 
pp. 215-216 e Tomassini 2012, pp. 221-224.

-2 Cfr. \#Brogi 1877; \#MPI 1879a; \#Direzione 1884;

\#Alinari 1885; \#Brogi 1889; \#Riproduzioni 1889.

$-{ }^{3}$ La ricerca svolta presso I'ASGF fa parte di una più ampia ricerca di dottorato sugli aspetti legali della riproduzione fotografica di opere d'arte e sul diritto d'autore per la fotografia. Ho fatto uno spoglio della documentazione conservata presso I'Archivio ricercando documenti riguardanti la fotografia (permessi, utilizzo, vendita e acquisto) per il periodo 1855-1915.

$-{ }^{4}$ Cfr. Edwards / Morton 2015, pp. 8-9 e

Edwards / Lien 2014, volumi nei quali il rapporto tra fotografia e musei è stato problematizzato e da cui questo saggio prende le mosse (cfr. anche Brusius 2013 e Sohier / Lugon / Lacoste 2017). La maggior parte della storiografia dedicata a questo argomento si è principalmente occupata di ricognizioni di materiale fotografico all'interno dei musei. Si differenziano, tra gli altri, per aver considerato anche le modalità di acquisizione $e$ di utilizzo della fotografia nei musei: Serena 2017; Paoli 2012; Ghibaudi 2012; Spiazzi / Majoli / Giudici 2010; Miraglia / Ceriana 2000. Un diverso discorso meritano alcuni studi sul rapporto tra Accademie di Belle Arti e fotografia, incentrati sui primi utilizzi della fotografia nell'insegnamento ma che hanno considerato anche le modalità di ingresso della fotografia nelle istituzioni. Per l'Accademia di Firenze cfr. Maffioli 2017,
Greco 2017, Migliorini 1994; per l'Accademia di Brera: Cassanelli 2015, Cassanelli 1996, Paoli 2012; per l'Accademia di Bologna: Giudici 2015; per l'Accademia di Venezia: Filippin 2015. In una prospettiva internazionale, mi limito qui a citare Hamber 1996; McCauley 1994; HaworthBooth / McCauley 1998. -5 Cfr. Berardi 2014, p. 185; Cestelli Guidi / Turco 2016, p. 47.

$-{ }^{6}$ Sul rapporto tra istituzioni centrali e periferiche cfr. Miraglia 2000.

-7 La bibliografia su questo tema è molto ricca, cfr. almeno: Caraffa 2011.

-8 La prima testimonianza di una raccolta di fotografie conservate presso il Gabinetto Disegni e Stampe si trova in \#Ferri 1883a. $-{ }^{9}$ Oltre al 'progetto Firenze', stando agli studi fino a oggi pubblicati, anche in altri musei le fotografie cominciarono a essere raccolte $\mathrm{e}$ collezionate (cfr. Miraglia 2000, p. 15, e Bonetti 2010). Escluso il caso del ricetto fotografico di Brera che prese forma negli anni Novanta dell'Ottocento (vedi Serena 2017, Ghibaudi 2012, Miraglia / Ceriana 2000), si tratta di progetti più tardi, ma sarebbe importante anche avere uno sguardo d'insieme sul fenomeno della costituzione di collezioni fotografiche presso le istituzioni museali.

$-{ }^{10}$ Sulle problematicità dell'archivio fotografico cfr. Schwartz 1995. Sul tema degli archivi fotografici delle soprintendenze cfr. Spiazzi / Majoli / Giudici 2010. Per quanto riguarda, invece, i concetti di collezione, raccolta, accumulo, sedimentazione, archivio e fotografia, cfr. Serena 2010.

- ${ }^{11}$ L'articolo di Chiara

Naldi pubblicato in questo stesso fascicolo (cfr. Naldi 2019), tratta dell'identificazione delle fotografie lasciate per deposito presso I'ASGF, mentre Arbeid 2015, esaminando le vicende dell'archivio fotografico del Museo Archeologico di Firenze, accenna alle fotografie lasciate per deposito. A completare il quadro fiorentino, Migliorini 1994 e più recentemente Maffioli 2017 hanno fatto riferimento all'utilizzo delle fotografie lasciate per deposito presso l'Accademia di Belle Arti di Firenze.

- 12 \#Alinari 1860. Le lettere sono trascritte in Quintavalle 2003, pp. 586-588. La richiesta Alinari del 1860 non fu la prima; già nel corso degli anni Cinquanta sia agli Alinari che a Eugène Piot fu concesso tale permesso, mentre ad altri fu rifiutato. Tuttavia, stando ai documenti conservati, questa è la prima occasione in cui si fa riferimento al lascito della copia. $-{ }^{13}$ Cfr. Filippin 2015, p.11 e Strambio 2000, p. 30. $-{ }^{14}$ Cfr. Migliorini 1994, p. 4. - ${ }^{15}$ Cfr. \#Ministero 1860e \#Pubblica Istruzione 1861. $-{ }^{16}$ Cfr. \#MPI 1867.

$-{ }^{17}$ A titolo esemplificativo, nelle quattro richieste avanzate dal 1861 al 1864 presso gli Uffizi non c'è nessun riferimento al lascito delle copie. - 18 \#Alinari 1860. $-{ }^{19} \mathrm{Cfr}$. Talk in the studio 1866 e McCauley 1994, p. 278. L'interdizione ai 
fotografi fu revocata nel 1872 quando fu introdotto il primo regolamento volto a disciplinare l'attività dei fotografi al Louvre, che prevedeva anche di lasciare due copie (Mathon / Pinet 2005, p. 14). Per la situazione inglese, cfr. Hamber 1996, p. 30 e

Brusius 2013, pp. 222-223. -20 RD 6 agosto 1893, n. 509; Legge 12 giugno 1902 n. 185. Per quanto riguarda i riferimenti legislativi in materia di riproduzioni di opere appartenenti allo Stato prima della legge del 1902, cfr. Giuseppe 1889, p. 121.

- 21 \#MPI 1867; \#MPI 1876; \#MPI 1877; \#MPI 1879b;

\#Norme 1881. La lista non è esaustiva sul piano nazionale.

- 22 I funzionari si

riferirono all'art. 5 del decreto sui calchi del 1873 che afferma che le spese occorrenti sono a carico del richiedente (RD n. 1727 Regolamento sui calchi delle opere d'arte) e agli art. 52 e 53 del decreto per il servizio del 1877 che affermano, rispettivamente, che per le riproduzioni dei monumenti restano ferme le prescrizioni del regolamento per i calchi $e$ che affinché un monumento possa estrarsi dalla collezione è necessaria la licenza del Ministro (RD n. 4359 Regolamento pel servizio dei musei d'antichità dello Stato). $-{ }^{23}$ Escluso il regolamento del 1881 che prevedeva che i fotografi potessero fotografare solo due opere alla volta (richiesta che non trovò quasi mai risposta per ragioni pratiche), $i$ regolamenti qui elencati presentano le stesse direttive.
-24 \#MPI 1876.

- 25 \#MPI 1878.

$-{ }^{26}$ Una questione

fondamentale, in relazione

all'utilizzo delle fotografie

a fini di studio, fu quella

dell'introduzione delle

lastre ortocromatiche, che

permettevano di restituire

una maggiore fedeltà

cromatica rispetto ai colori

del dipinto (cfr. Lavédrine

2009, p. 246 e Peters 2015, pp. 71-77).

-27 I resoconti riguardanti le difficoltà logistiche che si presentavano nel fotografare le opere d'arte nei musei si trovano in molte delle lettere conservate nell'ASGF e in Brogi 1885, pp. 9-10. Gli aspetti tecnici della riproduzione di opere sono stati considerati, tra gli altri, da Tomassini 2012, pp. 210-211.

- 28 Atti 1870-1874, p.

21. Gli Atti dell'inchiesta industriale sono citati in Tomassini 2012.

- 29 Alinari 1873, p. 9.

-30 Solo un sistematico riscontro tra le campagne fotografiche svolte dai fotografi e le fotografie conservate presso il fondo del MPI o presso i musei fiorentini, permetterebbe di affermare con certezza che i fotografi ottemperarono sempre alla richiesta delle copie. Tuttavia, ritengo verosimile che i fotografi abbiano generalmente lasciato le copie sia per i documenti citati in questo articolo attestanti il rilascio delle copie e il loro utilizzo, sia perché nei documenti conservati presso I'ASGF sono rare le occasioni di aperta contestazione tra la Direzione dei musei fiorentini e un fotografo per il non adempimento di tale richiesta.
- 31 \#Brogi 1877. Nel 1874,

Brogi aveva già lasciato

le copie all'Accademia di

Belle Arti di Firenze (cfr.

\#Brogi 1874).

- 32 \#Brogi 1874. Un altro

accenno polemico alla

questione risale al 1885 ,

quando nell'elencare le

difficoltà di riprodurre

opere d'arte nei musei,

il fotografo parla di "fare

regalo di copie" (Brogi

1885, p. 10).

- 33 Legge 25 giugno 1865

n. 2337. La fotografia non

era espressamente citata

come opera dell'ingegno

protetta dalla legge,

rendendo di conseguenza

il deposito potenzialmente

inutile. Nonostante ciò,

già negli anni Settanta,

la ditta Brogi fu una fra

le poche a presentare

alcune fotografie, seppure

in un numero limitato,

alla Prefettura di Firenze

per assicurarsi i diritti.

Sull'applicazione della

legge sui diritti d'autore alla

fotografia verte la mia tesi

di dottorato “Riproduzione

Interdetta. Photographs'

ownership and the

business of photographs

of artworks in Italy,

1860s-1920s". Sui depositi

di Brogi alla Prefettura di

Firenze negli anni Settanta,

vedi tra i depositi effettuati

Ministero di Agricoltura,

Industria e Commercio

1876, p. 3980.

$-{ }^{34}$ Sui progetti

ministeriali cfr. Mozzo

2018; Berardi 2014;

Marsicola 2014; Levi 2010.

$-{ }^{35}$ Cfr. \#Pigorini 1879a.

- 36 \#Pigorini 1879b.

-37 Ibid.

- 38 \#Pigorini 1879c.

- 39 Edwards / Lien 2014,

pp. 5-6.

- ${ }^{40}$ Cfr. \#Ferri 1883a.

-41 Ibid.

- ${ }^{42}$ Cfr. \#Donati 1881.

-43 Ibid. 
- 44 Cfr. \#Ferri 1883b;

\#Ferri 1884a; \#Ferri 1884b.

Le lettere scritte da Ferri

nel 1883 e nel 1884 sono

citate e in parte trascritte in Fileti Mazza 2014 (pp.

70-71), testo in cui vengono ricostruite le vicende del

Gabinetto.

- ${ }^{45}$ Cfr. \#Ferri 1887 e

\#Ferri 1890.

- 46 \#Ferri 1892.

- 47 \#Ridolfi 1895. Cfr. anche \#Progetto 1895.

- 48 \#Ridolfi 1895.

-49 lbid.

- 50 Per ragioni di spazio,

mi riferisco qui solo alla

collezione di fotografie

di dipinti e sculture,

escludendo dunque quelle

relative ai disegni.

- 51 Fileti Mazza 2014,

p. 40.

- 52 La collezione potrebbe essere stata smembrata

e le fotografie potrebbero

trovarsi disseminate tra $i$

vari nuclei conservati nel

Gabinetto delle Stampe,

ancora in attesa di essere

catalogate. Le fotografie

potrebbero anche essere

state spostate negli archivi

di altri musei fiorentini,

al tempo dipendenti dalla

Direzione delle Regie

Gallerie di Firenze, come

ad esempio il Museo

Archeologico di Firenze.

A ciò si aggiunge che un

nucleo di $\mathbf{4 8 0 0}$ fotografie

di proprietà di Nerino

Ferri furono acquistate dal

MPI e donate nel 1919 alla

Scuola di Storia dell'Arte di

Bologna (cfr. Calanna 2018,

p. 103 e Porcheddu 2008,

pp. 47-48).

-53 Per quanto riguarda il

ricetto di Brera, cfr. Serena

2017, Ghibaudi 2012,

Miraglia / Ceriana 2000.

- 54 RD 6 agosto 1893,

n. 509.

- 55 \#MPI 1889.

Per quanto riguarda

l'impatto della "profusion photographique" sulle modalità di collezionare fotografie e sul canalizzare anche fisicamente questo nuovo flusso di immagini da parte delle istituzioni museali, vedi Sohier / Lugon / Lacoste 2017, p. 9.

$-{ }^{56}$ RD 6 agosto 1893 ,

n. 509.

- 57 Tra i vari contributi

sull'argomento, cfr.

Marsicola 2014. In merito

agli altri progetti sulla

fotografia presentati

dal Ministero oltre

all'istituzione del

Gabinetto Fotografico

Nazionale, cfr. Mozzo 2018

e Serena 2015.

-58 Fusar Poli 2006, pp.

39-55.

- 59 Cfr. Circa la proposta

1892. Le tensioni tra

fotografi e Ministero sono

state in parte ricostruite da

Maffioli 2014, pp. 215-216

e Tomassini 2012, pp.

221-224.

- ${ }^{60}$ Alinari 1893, p. 6. II

libello di Vittorio Alinari fu pubblicato sul “Bullettino della Società fotografica italiana". Una copia si trova anche tra i documenti dell'ASGF.

- ${ }^{61}$ Ibid. Alinari aveva

criticato la disposizione di consegna dei negativi, ma questo riferimento non è chiaro perché il decreto stabiliva che il negativo fosse consegnato solo per i cimeli scientifici o letterari. È possibile che il fotografo si riferisse anche a una disputa, di cui però non sono chiari i termini, nella quale si trovava con la Direzione per non aver consegnato 44 negativi. - 62 \#Braun 1887.

- 63 \#Pieraccini 1888.

- ${ }^{64}$ Le nuove invenzioni

a cui sia Brogi che

Alinari fanno riferimento nelle loro lettere sono, probabilmente, le lastre ortocromatiche.

- ${ }^{65}$ Cfr. \#Pieraccini 1889

La lettera di Pieraccini portò alla sospensione di tutti permessi straordinari. - 66 Legge 12 giugno 1902 n. 185 e RD 7 novembre 1904, n. 431.

- ${ }^{67}$ RD 7 novembre 1904

n. 431, art. 249.

- 68 Atti Parlamentari

1904, p. 13746.

-69 Ivi, p. 13748.

- 70 Cfr. \#MPI 1904 e

Brogi 1904, pp. 13-16.

Fu questa sospensione

che portò l'onorevole

Filippo Torrigiani a

richiedere l'interpellanza

parlamentare prima

accennata (cfr. Atti

Parlamentari 1904).

Erano stati i fotografi ad avanzare tale richiesta come ricostruito in Per la protezione 1904b.

- 71 Cfr. McCauley 1994;

Renié 1999; Mathon / Pinet 2005.

- 72 Cfr. Brogi 1905 e

Brogi 1904, pp. 15-20. Sulle

reazioni dei fotografi cfr.

anche Maffioli 2014, pp.

215-216; Tomassini 2012,

pp. 221-224 e Binazzi

2020. Per quanto riguarda

la figura di Carlo Brogi e il

suo costante impegno nella tutela dei diritti dei fotografi cfr. Tomassini 1995.

$-{ }^{73}$ Cfr. Brogi 1904; Brogi 1905; Santoponte 1905, pp. 200-201.

-74 La Circolare è

citata da Brogi in Brogi 1904, p. 4, e in Tomassini 2012, pp. 221-224. Sia Giovanni Santoponte che Brogi sostennero, più o meno velatamente, che la sospensione dei permessi ai fotografi nel 1904 fosse stata una reazione del Ministero alla circolare emanata dalle aziende fotografiche (cfr. Santoponte 1905, p. 199, 
Brogi 1904, pp. 20-21, Per la protezione $1904 \mathrm{~b}, \mathrm{pp}$. 343-344).

- ${ }^{75}$ Santoponte 1905, pp. 200-201.

- 76 Brogi 1904, p. 8.

- 77 Cfr. \#Brogi 1905.

- 78 \#Brogi 1905.

- 79 RD 28 giugno 1906,

n. 447.

- 80 Cfr. \#Ricci [1903].

La trascrizione del documento si trova in

Tamassia 2011, pp. 73-74.

Per comprendere meglio il rapporto tra istituzioni centrali e periferiche sarebbe importante collegare l'appello di Ricci con la richiesta da parte delle istituzioni centrali di istituire gabinetti/archivi fotografici presso i musei come riferito da Miraglia 2000, p. 15 e da Serena 2017, p. 54.

- ${ }^{81}$ Cfr. Tamassia 2011.

- 82 Cfr. \#Soprintendente 1921 e Tamassia 2011. - 83 Cfr. \#Ferri 1908.
- 84 \#Parisotti 1911.

- 85 Per quanto riguarda lo status delle fotografie nei musei oggi, è possibile ricordare quanto affermato da Marina Miraglia sulla gestione dell'intero settore fotografia, che "appare lasciato fuori da una reale politica di tutela" (Miraglia 2000, p. 11; cfr. anche Edwards / Morton 2015).

Alinari 1873 Fratelli Alinari, Catalogo generale delle riproduzioni fotografiche pubblicate per cura dei Fratelli Alinari, Firenze, Tipografia di G. Barbera, 1873.

Alinari 1893 Vittorio Alinari, Del Regio Decreto e Regolamento per le riproduzioni fotografiche, in "Bullettino della Società fotografica italiana", n. 10, 1893, pp. 3-6.

Arbeid 2015 Barbara Arbeid, Un archivio completo di tutte le fotografie di scavi e di oggetti riguardanti le antichità dell'Etruria, in “Notiziario della Soprintendenza per i beni archeologici della Toscana", n. 11, 2015, pp. 1-8.

Atti 1870-1874 Atti del Comitato dell'inchiesta industriale. Deposizioni scritte. Categoria $13 \S 2$. Incisione Litografia e Fotografia, in Comitato dell'inchiesta industriale (a cura di), Atti del Comitato dell'Inchiesta Industriale (1870-1874). Deposizioni scritte, vol. I, Bologna, Analisi, 1984-1987, pp. 7-32.

Atti parlamentari 1904 Camera dei Deputati, Tornata di Lunedi 13 giugno 1904. Interrogazioni, in Atti parlamentari, Camera dei Deputati, sessione 19021904, Discussioni 7 giugno - 1 luglio 1904, Roma, Tipografia della Camera dei Deputati, 1904, pp. 13746-13748.

Berardi 2014 Elena Berardi, L'Archivio fotografico della direzione generale antichità e belle arti: genesi ed evoluzione del "fondo MPI", in “Bollettino d'arte", nn. 22-23, 2014, pp. 179-203.

Binazzi 2020 Marta Binazzi, Law and Canon. The Law's Influence on the Photographic Reproduction of Paintings Kept in the Florentine Royal Galleries, 1893-1904, in La reproduction des images et des textes/Images and Texts Reproduced, Leiden-Boston, Brill-Rodopi, 2020 [in corso di pubblicazione].

Bonetti 2010 Maria Francesca Bonetti, Il collezionismo fotografico nelle istituzioni: una necessità o una scelta?, in Francesco Faeta / Giacomo Daniele Fragapane (a cura di), Forme e modelli. La fotografia come modo di conoscenza, atti del convegno (Noto, 2010), Roma-Messina, Corisco Edizioni, 2010, pp. 53-62.

Brogi 1885 Carlo Brogi, In proposito della protezione legale sulle fotografie. Considerazioni di Carlo Brogi, Firenze, Tipografia Fratelli Bencini, 1885.

Brogi 1904 Carlo Brogi, In proposito del divieto fatto ai fotografi di trarre riproduzioni nei musei e gallerie dello Stato, Firenze, Tipografia di Enrico Ariani, 1904. 
Brogi 1905 Carlo Brogi, Memoriale presentato a S.E. il Ministro della Pubblica Istruzione a proposito delle condizioni imposte ai fotografi per riprodurre le opere d'arte e le antichità appartenenti allo Stato, Firenze, coi tipi di Enrico Ariani, 1905.

Brusius 2013 Mirjam Brusius, From Photographic Science to Scientific Photography: Talbot and Decipherment at the British Museum around 1850, in Mirjam Brusius / Katrina Dean / Chitra Ramalingam (a cura di), William Henry Fox Talbot. Beyond Photography, New Haven, Yale Center for British Art, 2013, pp. 219-244.

Calanna 2018 Giulia Calanna, II Fondo Supino oggi. Prospettive di ricerca a partire dalle testimonianze fotografiche di scultura pisana del Trecento di Alinari e Ammagliati, in "Intrecci d'arte Dossier," n. 4, 2018, pp. 102-121.

Caraffa 2011 Costanza Caraffa (a cura di), Photo Archives and the Photographic Memory of Art History, Berlin, Deutscher Kunstverlag, 2011.

Cassanelli 1996 Roberto Cassanelli, La fotografia nell'Accademia di Brera. Le prime acquisizioni, 1850-1860, in Marina Miraglia (a cura di), Luigi Sacchi lucigrafo a Milano 1805-1861, Milano, Federico Motta, 1996, pp. 31-38.

Cassanelli 2015 Roberto Cassanelli, Dalle aule al museo. La fotografia nelle accademie di Belle Arti nell'Ottocento, in Giovanna Cassese (a cura di), Patrimoni da svelare per le arti del futuro, atti del convegno (Napoli, 2013), Roma, Gangemi, 2015, pp. 212-221.

Cestelli Guidi / Turco 2016 Benedetta Cestelli Guidi / Simona Turco, Lo scavo ad isola Gorgo, Laguna di Grado, estate 1917. La documentazione visiva dell'archivio fotografico della ex Direzione Generale Antichità e Belle Arti, Ministero della Pubblica Istruzione, in “Quaderni Friuliani di Archeologia”, n. 1, 2016, pp. 47-56.

Circa la proposta 1892 Circa la proposta di colpire con una tassa le riproduzioni fotografiche dei monumenti nazionali, in "Bullettino della Società fotografica italiana", n. 4, 1892, pp. 101-103.

Edwards / Lien 2014 Elizabeth Edwards / Sigrid Lien, Museums and the Work of Photographs, in Elizabeth Edwards / Sigrid Lien (a cura di), Uncertain Images: Museums and the Work of Photographs, London-New York, Routledge, 2014.

Edwards / Morton 2015 Elizabeth Edwards / Christopher Morton, Between Art and Information: Towards a Collecting History of Photographs, in Elizabeth Edwards / Christopher Morton (a cura di), Photographs, Museums, Collections. Between Art and Information, London, Bloomsbury, 2015, pp. 3-23.

Fileti Mazza 2014 Miriam Fileti Mazza, Storia di una collezione. I disegni e le stampe degli Uffizi dal periodo napoleonico al primo conflitto mondiale, Firenze, Olschki, 2014.

Filippin 2015 Sara Filippin, “Questa fotografia non s'ha da fare...": Morris Moore, Raffaello e l'Accademia di Belle Arti di Venezia, in "RSF. Rivista di studi di fotografia", n. 1, 2015, pp. 8-25.

Fusar Poli 2006 Elisabetta Fusar Poli, La causa della conservazione del bello. Modelli teorici e statuti giuridici per il patrimonio storico-artistico italiano nel secondo ottocento, Milano, Giuffrè, 2006.

Ghibaudi 2012 Cecilia Ghibaudi, I/ ricetto fotografico della Pinacoteca di Brera: nascita e formazione di un archivio fotografico nell'Ottocento, in Maria Fratelli / Francesca Valli (a cura di), Musei nell'Ottocento, alle origini delle collezioni pubbliche lombarde, atti delle giornate di studio (Milano, 2010), Torino, Allemandi, 2012, pp. 425-435. 
Giudici 2015 Corinna Giudici, Un caso di collaborazione tra l'Accademia di Belle Arti di Bologna e la locale Soprintendenza per i Beni Storici e Artistici: esplorazioni della Fototeca Storica e spigolature, in Giovanna Cassese (a cura di), Patrimoni da svelare per le arti del futuro, atti del convegno (Napoli, 2013), Roma, Gangemi, 2015, pp. 207-211.

Giuseppe 1889 Francesco Giuseppe, Giur. Estera, vendita di un quadro, diritto di riproduzione, vendita allo Stato, tribunale di Parigi, $1^{\circ}$ marzo 1889, in "I diritti d'autore. Bollettino degli atti e notizie della società italiana degli autori", n. 11, 1893, pp. 120-122.

Greco 2017 Andrea Greco, Fotografia e documentazione d'arte all'Accademia di Belle Arti di Firenze 1839-1865, in Cristina Frulli / Francesca Petrucci (a cura di), L'Accademia di Belle Arti di Firenze negli anni di Firenze Capitale 1865-1870, atti del convegno (Firenze, 2015), Firenze, Edizioni dell'Assemblea, 2017, pp. 291-336.

Hamber 1996 Anthony Hamber, "A Higher Branch of the Art": Photographing the Fine Arts in England, 1838-1880, Australia-United Kingdom, Gordon and Breach, 1996.

Haworth-Booth / McCauley 1998 Mark Haworth-Booth / Anne McCauley, The Museum \& the Photograph. Collecting Photography at the Victoria and Albert museum, 1853-1900, Williamstown, Sterling and Francine Clark Art Institut, 1998.

Lavédrine 2009 Bernard Lavédrine, Photographs of the past. Process and preservation, Los Angeles, The Getty Conservation Institut, 2009.

Levi 2010 Donata Levi, Da Cavalcaselle a Venturi. La documentazione fotografica della pittura fra connoisseurship e tutela, in Spiazzi / Majoli / Giudici 2010, pp. 23-33.

Maffioli 2014 Monica Maffioli, Punti di vista: gli Stabilimenti fotografici italiani e il Gabinetto Fotografico di Giovanni Gargiolli, in Marsicola 2014, pp. 213-220.

Maffioli 2017 Monica Maffioli, "Del metodo del fare e del metodo del vedere": la fotografia all'Accademia di Belle Arti di Firenze nell'Ottocento, in Cristina Frulli / Francesca Petrucci (a cura di), L'Accademia di Belle Arti di Firenze negli anni di Firenze Capitale 1865-1870, atti del convegno (Firenze, 2015), Firenze, Edizioni dell'Assemblea, 2017, pp. 337-361.

Marsicola 2014 Clemente Marsicola (a cura di), Il viaggio in Italia di Giovanni Gargiolli. Le origini del Gabinetto Fotografico Nazionale 1895-1913, Roma, ICCD, 2014.

Mathon / Pinet 2005 Catherine Mathon / Helene Pinet, Les editeurs-photographes, in Monique Le Pelley Fonteny (a cura di), Adolphe \& Georges Giraudon. Une bibliothèque photographique, Paris-Somogy, Archives Départementales du Cher, 2005, pp. 12-17.

McCauley 1994 Elizabeth McCauley, Art Reproductions for the Masses, in Elizabeth McCauley, Industrial Madness. Commercial Photography in Paris 1848-1871, New Haven-London, Yale University Press, 1994, pp. 265-300.

Migliorini 1994 Chiara Migliorini, La fotografia come modello. L'Accademia di Belle Arti di Firenze, in "AFT. Rivista di Storia e Fotografia", n. 10, 1994, pp. 43-51.

Ministero di Agricoltura, Industria e Commercio 1876 Ministero di Agricoltura, Industria e Commercio, Elenco delle dichiarazioni e dei depositi iscritti nel registro del Ministero durante il mese di settembre 1876, per gli effetti delle leggi del 25 giugno 1865, numero 2337 e del 10 agosto 1875, n. 2652, in “Gazzetta Ufficiale del Regno d'Italia", n. 237, 1876, pp. 3979-3984.

Miraglia 2000 Marina Miraglia, La fortuna istituzionale della fotografia dalle origini agli inizi del Novecento, in Miraglia / Ceriana 2000, pp. 11-21. 
Miraglia / Ceriana 2000 Marina Miraglia / Matteo Ceriana (a cura di), Brera 1899, un progetto di fototeca pubblica per Milano. II "ricetto fotografico" di Brera, catalogo della mostra (Milano, Pinacoteca di Brera, 2000), Milano, Electa, 2000.

Mozzo 2018 Marco Mozzo, Base all'azione della Direzione Generale Antichità e Belle Arti. Note su una proposta di riforma di Adolfo Venturi, in "Annali di critica d'arte", n. 9, 2018, pp. 31-43.

Naldi 2019 Chiara Naldi, Le fotografie dell'Archivio Storico delle Gallerie Fiorentine (1875-1879), in “RSF. Rivista di studi di fotografia", n. 10, 2019, pp. 36-58.

Paoli 2012 Silvia Paoli, "Per il maggior incremento degli studi": la nascita delle raccolte fotografiche nei musei storico artistici di Milano, in Maria Fratelli / Francesca Valli (a cura di), Musei nell'Ottocento, alle origini delle collezioni pubbliche lombarde, atti delle giornate di studio (Milano, 2010), Torino, Allemandi, 2012, pp. $425-435$.

Peters 2015 Dorothea Peters, ... der allerböseste Punkt: die Suche nach dem richtigen Tonwert, in Cornelia Kemp (a cura di), Unikat Index Quelle. Erkundungen zum Negativ in Fotografie und Film, Göttingen, Wallstein Verlag, 2015, pp. 61-83.

Porcheddu 2008 Giorgio Porcheddu, La Fototeca del Dipartimento delle Arti Visive: memoria, ricerca, didattica, in "Acta Photographica", n. 1, 2008, pp. 41-54.

Per la protezione 1904a Per la protezione dei diritti dei fotografi, in "Bullettino della Società fotografica italiana", n. 6, 1904, pp. 201-204.

Per la protezione 1904b Per la protezione dei diritti dei fotografi, in "Bullettino della Società fotografica italiana", n. 10, 1904, pp. 343-344.

Quintavalle 2003 Arturo Carlo Quintavalle, Gli Alinari, Firenze, Alinari, 2003.

Renié 1999 Pierre-Lin Renié, Braun versus Goupil et quelques autres histories. La photographie au musée du Louvre au XIX siècle, in Pierre-Lin Renié (a cura di), État des lieux, Bordeaux, Musée Goupil, 1999, pp. 97-152.

Santoponte 1905 Giovanni Santoponte, Questioni professionali, in "Annuario della fotografia e delle sue applicazioni", a. VI, parte IV, 1905, pp. 199-201.

Schwartz 1995 Joan M. Schwartz, "We make our tools and our tools make us": Lessons from Photographs from the Practice, Politics and Poetics of Diplomatics, in "Archivaria", vol. 40, 1995, pp. 40-74.

Serena 2010 Tiziana Serena, L'archivio fotografico: possibilità derive potere, in Spiazzi / Majoli / Giudici 2010, pp. 103-125.

Serena 2015 Tiziana Serena, Cultural Heritage, Nation, Italian State: Politics of the Photographic Archive Between Centre and Periphery, in Costanza Caraffa / Tiziana Serena (a cura di), Photo Archives and the Idea of Nation, Berlin, De Gruyter, 2015, pp. 179-200.

Serena 2017 Tiziana Serena, Le musée d'art comme lieu d'autorité pour l'archive photographique. Le cas italien au tournant du XX siècle, in Musées de photographies documentaires, num. mon. di “Transbordeur", n. 1, 2017, pp. 50-61.

Sohier / Lugon / Lacoste 2017 Estelle Sohier / Olivier Lugon / Anne Lacoste, Introduction. Les collections de photogrpahies documentaires au tournant du XX siècle, in Musées de photographies documentaires, num. mon. di “Transbordeur", n. 1, 2017, pp. 8-17.

Spiazzi / Majoli / Giudici 2010 Anna Spiazzi / Luca Majoli / Corinna Giudici (a cura di), Gli archivi fotografici delle soprintendenze. Tutela e storia, atti del convegno (Venezia, 2008), Crocetta del Montello, Terra Ferma, 2010. 
Strambio 2000 Andrea Strambio, Profilo documentario della Fototeca di Brera, in Miraglia / Ceriana 2000, pp. 29-35.

Talk in the studio 1866 Talk in the Studio. Photography in the Louvre, in "The Photographic News", n. 419, 1866, p. 443.

Tamassia 2011 Marilena Tamassia, Primi anni di attività del Gabinetto Fotografico 1904-1919, Livorno, Sillabe, 2011.

Tomassini 1995 Luigi Tomassini, Le origini della Società Fotografica Italiana e lo sviluppo della fotografia in Italia. Appunti e problemi, in "AFT. Rivista di Storia e Fotografia", n. 1, 1995, pp. 42-51.

Tomassini 2012 Luigi Tomassini, La costruzione dell'immagine fotografica dell'Italia unita, fra pubblico e privato: i grandi fotografi editori del XIX secolo, in Andrea Ragusa (a cura di), La nazione allo specchio. Il bene culturale nell'Italia unita, 1861-2011, Manduria, Lacaita, 2012, pp. 193-224.

\#Alinari 1860 Fratelli Alinari, Eccellenza..., lettera ms., Firenze (d'ora in poi Fi), 18 giugno 1860. Fi, Archivio Storico delle Gallerie Fiorentine (d'ora in poi ASGF), 1860, LXXXIV, 51.

\#Alinari 1885 Stabilimento Fotografico dei Fratelli Alinari, Sig. R.R. Gallerie..., lettera ms., Fi, 6 maggio 1885. Fi, ASGF, 1885, J, Affari Generali, 32.

\#Braun 1887 Ad. Braun et Cie, Eccellenza..., lettera ms., Dornach, 13 dicembre 1887. Fi, ASGF, 1887, F, Affari Generali, 3.

\#Brogi 1874 Giacomo e Carlo Brogi, In conformità..., lettera ms., Fi, 13 agosto 1874. Fi, Archivio Storico dell'Accademia di Belle Arti di Firenze, 1874, 63, 13.

\#Brogi 1877 Giacomo e figlio Brogi, Conforme..., lettera ms., Fi, 14 febbraio 1877. Fi, ASGF, 1877, A, Galleria delle Statue, 31.

\#Brogi 1889 Giacomo Brogi Fotografo Editore, Signor Direttore..., lettera ms., Fi, 6 novembre 1889. Fi, ASGF, 1889, E, Affari Generali, 1.

\#Brogi 1905 Carlo Brogi, Egregio Caro..., lettera ms., Fi, 26 dicembre 1905. Fi, ASGF, 1904, 325, Direzione, 51.

\#Direzione 1884 Direzione, Mi reco a..., lettera ms., Fi, 19 febbraio 1884. Fi, ASGF, 1884, Galleria degli Uffizi, 7.

\#Donati 1881 Cesare Donati, Prima che..., lettera ms., Fi, 21 ottobre 1881. Fi, ASGF, 1881, D, Galleria degli Uffizi, 274.

\#Ferri 1883a Nerino Ferri, Nota..., lettera ms., Fi, 8 maggio 1883. Fi, ASGF, 1883, D, Direzione, 62.

\#Ferri 1883b Nerino Ferri, Seconda..., lettera ms., Fi, 3 agosto 1883. Fi, ASGF, 1883, D, Direzione, 62.

\#Ferri 1884a Nerino Ferri, Appendice $3^{\circ}$..., lettera ms., Fi, 11 gennaio 1884. Fi, ASGF, 1884, E, Galleria degli Uffizi, 7.

\#Ferri 1884b Nerino Ferri, Nota..., lettera ms., Fi, 30 gennaio 1884. Fi, ASGF, 1884, E, Galleria degli Uffizi, 7.

\#Ferri 1887 Nerino Ferri, Nota..., lettera ms., Fi, 3 agosto 1887. Fi, ASGF, 1887, F, Affari Generali, 3.

\#Ferri 1890 Nerino Ferri, Nota..., lettera ms., Fi, 29 gennaio 1890. Fi, ASGF, 1890, Affari Generali, 5.

\#Ferri 1892 Nerino Ferri, Accuso ricevimento..., lettera ms., Fi, 22 novembre 1892. Fi, ASGF, 1892, C, Affari Generali, 6. 
\#Ferri 1908 Nerino Ferri, Stimo opportuno..., lettera ms., Fi, 27 giugno 1908. Fi, ASGF, 1908, Gabinetto Disegni e Stampe, 1.

\#Ministero 1860 Ministero della Pubblica Istruzione (d'ora in poi MPI), Regolamento..., lettera ms., Fi, 13 marzo 1860. Fi, ASGF, 1860, LXXXI, 19.

\#MPI 1867 MPI, Vengono a..., lettera ms., Fi, 14 ottobre 1867. Fi, ASGF, 1867, A, Galleria delle Statue, 107.

\#MPI 1876 MPI, Circolare..., lettera ms., Roma, 23 giugno 1876. Fi, ASGF, 1876, A, Galleria delle Statue, 84.

\#MPI 1877 MPI e Direzione delle RR. Gallerie di Firenze, Norme..., mat. a stampa, poster e libro, $1^{\circ}$ agosto 1877. Fi, ASGF, 1877, B, Galleria delle Statue, 124.

\#MPI 1878 MPI, Visto il parere..., lettera ms., Roma, $1^{\circ}$ febbraio 1878. Fi, ASGF, 1878, A, Galleria delle Statue, 13.

\#MPI 1879a MPI, Ho ricevuto..., lettera ms., Roma, 26 marzo 1879. Fi, ASGF, 1879, B, Galleria delle Statue, 93.

\#MPI 1879b MPI e Direzione delle RR. Gallerie di Firenze, Norme..., mat. a stampa, poster, Fi, 7 aprile 1879. ASGF, 1879, A, Gallerie delle Statue, 37.

\#MPI 1889 MPI, Circolare..., lettera ms., Roma, 19 settembre 1889. Fi, ASGF, 1889, E, Affari Generali, 1.

\#MPI 1904 MPI, Circolare..., lettera dattil., Roma, 8 giugno 1904. Fi, ASGF, 1904, Direzione, 23.

\#Norme 1881 Norme..., Firenze, Tipografia Mazzocchi, 1881, mat. a stampa. Fi, ASGF, 1881, B, Galleria degli Uffizi, 97.

\#Parisotti 1911 Alberto Parisotti, Nella mia..., lettera dattil., [?] dicembre 1908. Fi, ASGF, 1911, Gabinetto Disegni e Stampe, 1.

\#Pieraccini 1888 Eugenio Pieraccini, Condizioni..., lettera ms., s.l., s.d. Fi, ASGF, 1888, F, Affari Generali, 2.

\#Pieraccini 1889 Eugenio Pieraccini, Al Direttore..., lettera ms., Fi, 18 settembre 1889. $\mathrm{Fi}$, ASGF, 1890, Affari Generali, 5.

\#Pigorini 1879a Luigi Pigorini, Mi pregio..., lettera ms., Fi, 20 marzo 1879. Fi, ASGF, 1879, B, Galleria delle Statue, 93.

\#Pigorini 1879b Luigi Pigorini, Per rispondere..., lettera ms., Fi, 26 marzo 1879. Fi, ASGF, 1879, B, Galleria delle Statue, 103.

\#Pigorini 1879c Luigi Pigorini, Per corrispondere..., lettera ms., Fi, 17 aprile 1879. Fi, ASGF, 1879, B, Galleria delle Statue, 103.

\#Progetto 1895 Progetto di impianto di un'Amministrazione relativa alle riproduzioni fotografiche dovute dai Signori fotografi alla Direzione e al Ministero, lettera ms., s.l., s.d. Fi, ASGF, 1895, C, Affari generali, 4.

\#Pubblica Istruzione 1861 Direzione segreteria della Pubblica Istruzione, Regolamento..., lettera ms., Fi, 25 marzo 1861. Fi, ASGF, 1861, 39.

\#Ricci [1903] Corrado Ricci, Archivio Fotografico, mat. a stampa, timbro del 16 novembre 1903. Fi, ASGF, 1904, Direzione, 41.

\#Ridolfi 1895 Enrico Ridolfi, Questa direzione..., lettera ms., Fi, 22 novembre 1895. Fi, ASGF, 1895, C, Affari generali, 4.

\#Riproduzioni 1889 Riproduzioni fotografiche..., varie lettere ms., Fi, ASGF, 1889, E, Affari Generali, 1.

\#Soprintendente 1921 II Soprintendente, Allegato..., lettera dattil. e a stampa, Fi, 21 dicembre 1921. Fi, ASGF, 1921, Gabinetto Fotografico, 1. 